Onkologe 2010 · 16:223-224

DOI 10.1007/s00761-010-1807-9

Online publiziert: 4. März 2010

(c) Springer-Verlag 2010

\author{
H. Ludwig ${ }^{1}$ K. Höffken \\ ${ }^{1} 1$. Medizinische Abteilung, Zentrum für Onkologie \\ und Hämatologie, Wilhelminenspital, Wien \\ ${ }^{2}$ Universitätsklinikum Jena
}

\title{
Multiples Myelom: neue Perspektiven für einen kurativen Therapieansatz
}

Das multiple Myelom ist eine Erkrankung mit sehr unterschiedlichem Krankheitsverlauf, was auf die beträchtliche zytogenetische und molekulargenetische $\mathrm{He}$ terogenität zurückzuführen ist. Im vergangenen Jahr konnte der Nachweis erbracht werden, dass alle oder fast alle $\mathrm{Pa}$ tienten vor Manifestation eines multiplen Myeloms ein MGUS-Stadium durchlaufen. Im Verlauf der Erkrankung kommt es zu einer zunehmenden genetischen Instabilität mit beispielsweise Deaktivierung von Tumorsuppressorgenen und Aktivierung von Genen, die Transkriptionsfaktoren kodieren. Die Transformationsrate vom MGUS in ein multiples Myelom liegt bei $1 \%$ pro Jahr. Zytogenetische Parameter wie Translokationen von Chromosom 14, Deletion von 17p und Veränderung an lq21 kommt wichtige prognostische und prädiktive Bedeutung zu. Obwohl zytogenetische Veränderungen (mit Ausnahme von del 17p) bei Patienten mit MGUS und Myelom in ähnlicher Häufigkeit nachweisbar sind, finden sich deutliche Unterschiede in Genexpressionsprofilen. Unklar bleibt weiterhin, welche Mechanismen letztendlich zur Transformation in ein multiples Myelom fuhren.

Auf diagnostischem Gebiet konnte durch die Einführung des Freie-Leichtketten-Tests die Diagnose und Verlaufsbeurteilung von Patienten mit nicht- oder wenig sezernierendem Myelom sowie mit Amyloidose verbessert werden. Zum Nachweis einer minimalen Resterkrankung eignet sich eine 6-8-Farben-Immunphänotypisierung oder PCR, wobei mit erstgenannter Methode bereits eine Sensitivität von 1:10 ${ }^{6}$ erreicht wird. Derzeit wird die Skelettradiographie noch als Standardmethode zum Nachweis myelomspezifischer Läsionen betrachtet. Allerdings kann mit den modernen bildgebenden Verfahren wie MRT, Ganzkörper-MRT, PET und PET-CT eine größere diagnostische Sensitivität erreicht werden; ferner dürfte ein posttherapeutischer PET-Befund prognostisch relevant sein.

\section{( Der überlegene Stellenwert sogenannter neuer Substanzen ist mittlerweile bewiesen}

In den letzten Jahren konnte der überlegene Stellenwert der sogenannten neuen Substanzen Thalidomid, Bortezomib und Lenalidomid verglichen mit konventionellen Zytostatika in zahlreichen Studien unter Beweis gestellt werden. Mit modernen Induktionsprotokollen können weitaus höhere Ansprechraten bereits vor und insbesondere nach Hochdosistherapie mit anschließender autologer Stammzelltransplantation erreicht werden. Diese führen zu einer signifikanten Verlängerung des progressionsfreien Überlebens. Ob dadurch auch eine Verlängerung des Gesamtüberlebens möglich wird, kann derzeit noch nicht beantwortet werden. Bei jüngeren Patienten ( $<65$ Jahre) gilt die Hochdosistherapie mit autologer Stammzelltransplantation nach wie vor als Standardtherapie.

Zurzeit wird der Frage nachgegangen, ob mit den neuen Therapien allei- 
ne gleich gute Ergebnisse wie mit hoch dosierter Chemotherapie mit autologer Stammzelltransplantation in Kombination mit den neuen Substanzen erzielt werden können.

Bei älteren Patienten konnte die Wirksamkeit von Melphalan/Prednison durch die Kombination mit Thalidomid oder Bortezomib deutlich gesteigert werden, insbesondere auch im Hinblick auf die Überlebensraten. Neuere Studien haben eine hohe Toxizität von hoch dosiertem Dexamethason in Kombination entweder mit Thalidomid oder Lenalidomid v. a. bei älteren Patienten mit schlechtem Allgemeinzustand aufgezeigt, was zu einem $\mathrm{Pa}$ radigmenwechsel der Dexamethasontherapie geführt hat. Niedrig dosiertes Dexamethason plus Lenalidomid ist der Kombination von konventionell hoch dosiertem Dexamethason mit Lenalidomid deutlich überlegen und führt zu besseren Therapieergebnissen und geringerer Toxizität.

Gegenwärtige Bemühungen konzentrieren sich auf die Optimierung der Therapieergebnisse durch Konsolidierungsbzw. Erhaltungstherapien mit Lenalidomid- und Bortezomibkombinationen. Für die Lenalidomiderhaltungstherapie liegen bereits Hinweise für eine signifikante Verlängerung der progressionsfreien Überlebenszeit vor. Von den zahlreichen neuen Substanzen, die derzeit in klinischen Studien überprüft werden, zeigen der Proteasominhibitor Carfilzomib sowie das IMiD Pomalidomid hohe klinische Wirksamkeit. Insgesamt ist es durch die Einführung der neuen Substanzen gelungen, die Überlebenszeit bei jüngeren Patienten deutlich zu verlängern.

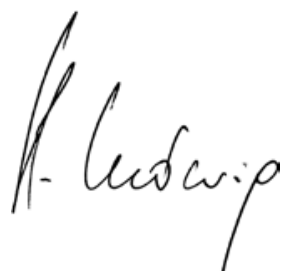

Heinz Ludwig

Für die Herausgeber des Schwerpunktheftes

\section{Hörtren}

Klaus Höffken

Für die Herausgeber

\section{Korrespondenzadresse}

Prof. Dr. H. Ludwig

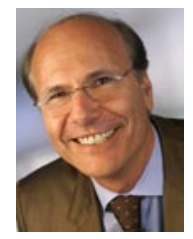

1. Medizinische Abteilung, Zentrum für Onkologie und Hämatologie, Wilhelminenspital Montleartstraße 37, 1160 Wien Österreich heinz.ludwig@wienkav.at

\section{Neue Fortschritte bei Angiogeneseforschung}

Einen wichtigen Schritt zum besseren Verständnis der molekularen Vorgänge bei der Angiogenese von Tumoren haben nun Forschende des Paul Scherrer Instituts und des Biomedicum in Helsinki, Finnland gemacht. Sie haben die Struktur der Bindungsstelle aufgeklärt, an der sich ein Wachstumsfaktor mit dem entsprechenden Rezeptor auf der Zelloberfläche verbindet. Durch diese Verbindung wird das Wachstum der Lymphgefäße angeregt. Das von den Forschern untersuchte Molekül ist der VEGF-C (vascular endothelial growth factor- $\mathrm{C}$ ), dessen Bindung an den Rezeptor die Zelle angeregt, sich zu teilen und so am Wachstum neuer Blut- und Lymphgefäße teilzuhaben.

Um die Proteinstrukturen von Ligand und Rezeptor im Detail zu bestimmen, haben die Forscher die Moleküle mittels Proteinkristallographie an der Synchrotron Lichtquelle Schweiz SLS des Paul Scherrer Instituts untersucht.

Dieses Wissen kann in der Zukunft für die weitere Entwicklung neuer Medikamente verwendet werden, die diese Rezeptoren gezielt blockieren und so das Wachstum der Gefäße verhindern.

Literatur: Leppänen VM, Prota $A E$, Jeltsch Met al (2010) Structural determinants of growth factor binding and specificity by VEGF receptor 2. PNAS Early Edition, January 22

Quelle: Paul Scherrer Institut, www.psi.ch 